\title{
Implicit Cognition for Alcohol in the Workplace: An Essay Review Article for Health Promotion and Human Resources Management
}

\author{
Keitiline R. Viacava \\ Federal University of Rio Grande do Sul (UFRGS), Brazil
}

Received: Nov. 2, 2016

Accepted: Nov. 27, $2016 \quad$ Published: January 1, 2017

doi:10.5296/jmr.v9i1.10248

URL: http://dx.doi.org/10.5296/jmr.v9i1.10248

\begin{abstract}
Alcohol misuse is an occupational risk factor and, therefore, a regular theme in health-promotion programs in the workplace. However, Human Resources Management could expand the effectiveness of these programs, especially if implicit measures, such as attentional bias for alcohol cues were assessed. Attentional bias is an automatic behavior, which is the tendency to direct attention to emotionally relevant stimuli (e.g., pleasure, reward, danger). It was previously observed in alcohol-dependent (and non-dependent) adults and appointed as a risk marker to alcohol misuse. Thus, the aim of this paper is to theoretically address possible relationships between frequency of participation in alcohol-prevention programs in the workplace and attentional bias to alcohol cues related to drinking. We suggest that implicit measures of risk for alcohol misuse could be assessed by the organizations and that lower results could be found in employees with frequent participation in alcohol-prevention programs at work. This discussion can contribute to definitions about frequency schedules for preventive actions at work and can support the development of cognitive-oriented technologies to identify employees' implicit motivational states in relation to health goals.
\end{abstract}

Keywords: Alcohol, Occupational Risk, HRM, Implicit Measures, Attentional Bias 


\section{Introduction}

Unhealthy living habits, such as excessive consumption of alcohol, are associated with high rates of absenteeism, low productivity, and poor performance at work (Nigatu, Reijneveld, Penninx, Schoevers, \& Bultmann, 2015; Rongen, Robroek, van Lenthe, \& Burdorf, 2013). For this reason, and also because of the organizational context being considered favorable for carrying out targeted interventions to reduce these problems, health-promotion activities are commonly performed by Human Resources Management (HRM) (Aldana, Merrill, Price, Hardy, \& Hager, 2005; Bazzani \& Sanchez, 2016). However, recent research has provoked reflections on the scope of these interventions(Rongen et al., 2013), as a Swedish study focused on conscious consumption of beverages by public servants and revealed only low to moderate results (Tinghog, 2014; Tinghog \& Tinghog, 2016). Understanding how these programs affect the drinking behavior of employees is a relevant issue, especially when considering health as an occupational risk factor (Cabecas, 2015).

Factors that encourage the HRM to control the misuse of alcohol include the prevention of aggressive behavior at work (Barling, Dupre, \& Kelloway, 2009), reducing abusive supervisory practices(Byrne et al., 2014) and insubordination (Spicer \& Miller, 2016), the risk of suicide (Balsa, French, \& Regan, 2014; Eliason \& Storrie, 2009), the containment of prejudice and the social stigma attached to drinking (Gedro, Mercer, \& Iodice, 2012), and the reduction of medium- and long-term health care costs (Deitz, Cook, \& Hersch, 2005).

Periods of crisis and unemployment may present an additional reason for carrying out actions to control the consumption of alcohol, especially if we consider the data showing that the economic downturn was positively related to frequency of use and alcohol poisoning in middle-aged male and female American workers (Frone, 2016). Some possible determinants of the risk for alcohol misuse include such factors as gender (with the highest prevalence found in men), individual aspects (depression, traumatic events), social norms at work (permissive practices to drink), working conditions (overload, stress, little collegiality), low education, and low income (Roche et al., 2015).

One possible explanation for obtaining moderate (or even low) results in alcohol-prevention programs may be because these actions are developed after a behavior model based mainly on planned cognitive processes. This trend can be seen in the emphasis given to the provision of sporadic or isolated information about the potential benefits to employees in controlling or changing their drinking behaviors. By pegging behavior change to future consequences, the success of interventions is subject to the planning capacity of individuals; that is, it is almost exclusively dependent on explicit, controlled processes, which could impair the results (Marteau, Hollands, \& Fletcher, 2012).

It is believed that the effectiveness of the programs depends on the recognition of implicit, automatic processes that involve behavior and focused interventions, with frequent exposure to associations geared to healthy habits (Marteau et al., 2012). The rationale for this approach lies in the set of accumulated evidence, indicating that much information processing takes place outside of the reach of consciousness, being unintentional, independent of goals, and guided by habit and associative learning (Moors \& De Houwer, 2006). Therefore, this study 
is based on the idea that organizations can reduce the risk of alcohol misuse by its employees through health-promotion programs, thus creating a healthy work environment. However, considering that this result is subject to change implicit processes related to drinking, we suggest that implicit measures of risk for alcohol misuse could be assessed by the organizations and that lower results could be found in employees with frequent participation in alcohol-prevention programs at work. Therefore, the aim of this paper is to theoretically address possible relationships between frequency of participation in alcohol-prevention programs in the workplace and attentional bias to alcohol cues related to drinking.

On a theoretical basis, we suggest the use of a multidisciplinary approach, which includes studies derived from the fields of HRM, Cognitive Psychology, Experimental Psychology, and Additions. Essentially, this study is anchored in the dual-process model of information processing (Hofmann, Gschwendner, Friese, Wiers, \& Schmitt, 2008), although it adopts a more relative view of the distinction between implicit and explicit processes (Cohen, Dunbar, \& McClelland, 1990; Moors \& De Houwer, 2006). In particular, this essay welcomes the suggestions presented in the 23rd edition of the influential Human Resources Management Reviewin2013 with respect to the use of implicit measures in HRM; this choice was particularly influenced by the study entitled Digging deeper or piling it higher? Implicit measurement in organizational behavior and human resource management (Haines \& Sumner, 2013).

\section{Cognitive Implicit Measures in HRM}

To advance the understanding of the influence of automatic motivational factors, researchers have drawn attention to the inclusion of implicit measures in research on HRM and organizational behavior(Barsade, Ramarajan, \& Westen, 2009; Becker \& Menges, 2013; Becker \& Cropanzano, 2010; Becker, Cropanzano, \& Sanfey, 2011; Cropanzano \& Becker, 2013; Haines \& Sumner, 2013). These suggestions are based on advances in the field of psychology, in particular on findings that human behavior is not always deliberate, reflective, flexible, and motivated by future consequences, but that it is often automatic, implicit, poorly controlled, and guided by context (Marteau et al., 2012; Moors \& De Houwer, 2006). Implicit measures are generally obtained from reaction-time studies; however, its use is not exempt from criticism and has potentials and limitations (Haines \& Sumner, 2013).

If the implicit measures offer the opportunity to capture spontaneous responses, which are effective in routine situations and not without awareness, then, conversely, they impose the challenge of defining what to do with this dimension of human behavior in terms of HRM. Adopting a more pragmatic point of view, it is argued about the usefulness of these assessments in management, because they are considered rigid, not conscious, and difficult to change. One of the reasons for this concern can be supported in the model that anchors the distinction between implicit and explicit processes; this is the dual-process model of information processing (Hofmann et al., 2008).

According to this model, observed behaviors in response to stimuli presented for times less than half a second are interpreted as implicit (not aware), whereas longer response times are considered explicit (conscious). Although the dichotomous distinction between implicit and 
explicit is claimed to be more didactic than realistic (Cohen et al., 1990; Moors \& De Houwer, 2006), it is relatively convergent with consciousness studies (Block, 2005), which can sustain some skepticism about the modification possibilities of implicit behaviors.

There is, however, an alternative and more flexible view of this distinction, which considers the behavior as continuous (rather than all or nothing), able to move from implicit to explicit, and vice versa (Cohen et al., 1990; Moors \& De Houwer, 2006). For example, it is accepted that, with time, effort, and motivation, individuals can evolve from implicit to explicit behaviors (Cunningham, Zelazo, Packer, \& Van Bavel, 2007; Viacava, Weydmann, Tietze, Santolim, \& Bizarro, 2016). The opposite is also admitted, but the transition from explicit to implicit behavior seems to depend on repeated exposure to new associations (Lopes, Viacava, \& Bizarro, 2015).

\section{Health Promotion by Mere-Exposure}

According to the mere-exposure effect, it is accepted that individuals may develop new preferences, depending on the frequency of exposure, even if they cannot report explicitly (Zajonc, 1968). This supports the previously mentioned hypothesis that repeated participation in alcohol-prevention programs at work can cause changes in the drinking behaviors of employees. An important feature of this effect is that it can be interpreted in terms of a continuum between habituation and boredom. Whereas habituation may influence the behavior by familiarity, boredom triggered by overexposure can reduce and even extinguish the exposure effect (Bornstein, 1989; B. M. Robinson \& Elias, 2005). In addition, the relationship between frequency and exposure seems to be quadratic (non-linear), and larger effects should be observed between the beginning and the end of the habituation phase, declining at the boring phase (Bornstein, 1989; B. M. Robinson \& Elias, 2005).

This idea is particularly relevant for this essay because it highlights a possible relationship between frequency of exposure and effect of the intervention. If observed empirically, it can support the planning of health programs at work, influencing frequency schedule settings, for example. Organizations could focus their preventive actions on the employees' early years with the company and reduce them over time, focusing its investments at the stage of habituation. Another interesting feature of the mere-exposure effect is that it can be modulated by selective attention (Yagi, Ikoma, \& Kikuchi, 2009); a previous study showed that not only repetition frequency, but also attention, can influence preferences and attitudes (Huang \& Hsieh, 2013). This feature can be especially useful for the creation of performance indicators or effectiveness measures of health interventions by HRM.

Following this direction, recent research has focused on the role of selective attention on safety at work (Xu et al., 2014). Converging closely with this discussion, the authors used two models based on reaction time to measure attentional bias. Their results revealed that employees with better security behavior were more likely to direct attention to safety-related stimuli as compared with employees with unsatisfactory safety behaviors. They also demonstrated that attention was positively associated with motivation and the security climate perceived by employees. This research contributed to the understanding of the early stages of implicit perceptual processing involved in safety behaviors. However, unlike 
previous research that focused on implicit attitudes (Burns, Mearns, \& McGeorge, 2006; Marquardt, Gades, \& Robelski, 2012; Molesworth \& Chang, 2009), this study introduced implicit measures of attention in the understanding of employees' basic psychological processes.

\section{Implicit Measures of Attentional Bias}

The relevance of discussing the relationship between participation in alcohol-prevention programs and the selective attention of employees is based on the accumulated evidence showing associations between the direction of attention to stimuli related to alcohol and alcohol misuse, and these were observed in alcohol-dependent (and non-dependent) adults (Cox, Brown, \& Rowlands, 2003; Field \& Cox, 2008). The tendency to select potentially important stimuli (positive or negative) as a reward, pleasure, satiety (di Pellegrino, Magarelli, \& Mengarelli, 2011), or danger (Williams, Mathews, \& MacLeod, 1996), to the detriment of other environmental stimuli, is known as attentional bias. Attentional bias is an automatic behavior (Stormark, Laberg, Nordby, \& Hugdahl, 2000)that can be affected by several factors, such as mood (Hepworth, Mogg, Brignell, \& Bradley, 2010), stress(Field \& Quigley, 2009), self-esteem (Li, Yang, Jia, \& Zhang, 2011), and sensitivity to reward.

Attentional bias is widely known in the literature as a behavioral risk marker to alcohol misuse or predisposition to addiction (Dager et al., 2014; Field \& Cox, 2008; Robbins \& Ehrman, 2004). It is believed that alcohol cues can capture attention because they trigger a cascade of neural events involved in reward processing as motivated behavior influencing the approach or avoidance of stimuli (Field \& Cox, 2008). These events can lead to increased relevance being attached to the item to which attention was allocated by increasing the desire for it and promoting its consumption (T. E. Robinson \& Berridge, 1993).

Attentional bias measurements can be obtained from different tasks and methods (Field, Munafo, \& Franken, 2009). The most traditional is the modified version of the Classic Stroop(Stroop, 1992) or Emotional Stroop (Williams et al., 1996). During the task, participants are instructed to quickly identify the color in which the words (e.g., emotionally relevant or neutral) are printed and try to ignore the semantic content of the words. Attentional bias is measured from the reaction time for naming the words. It is assumed that slower reaction times reflect higher interference being generated by the words (Cox, Fadardi, \& Pothos, 2006; Field et al., 2009). The Emotional Counting Stroop (ecStroop) is an adapted version of the Emotional Stroop (Bush, Whalen, Shin, \& Rauch, 2006; Whalen, Bush, Shin, $\&$ Rauch, 2006). In this task, the words are displayed vertically on the screen, and participants are asked to press the number key corresponding to the number of times the word was listed. The Stroop task was recently appointed as an implicit measure to support the selection and performance evaluation of employees in positions that require high emotional control, such as those related to customer service and telesales (Becker, Volk, \& Ward, 2015).

Another widely used measure of attentional bias is the Visual Probe Task, in which a small symbol is hidden by a target image (e.g., alcohol) or a control image (e.g., neutral object), both simultaneously displayed on the computer screen. The control target images disappear, 
and the symbol (which may be a dot, an arrow, letters, or geometric figures) is displayed, replacing the position occupied by one of the two previous images. Participants are commonly instructed to respond to the position of the symbol as soon as possible, and the reaction times for the conditions in which the symbol was hidden by the target images are compared with the reaction times for conditions in which they symbol was hidden by control images (Field et al., 2009).

The Visual Probe Task was originally developed for anxiety studies (Macleod, Mathews, \& Tata, 1986), based on the idea that people tend to respond more quickly to stimuli presented in the area to which attention was previously allocated (Navon \& Margalit, 1983; Posner, Snyder, \& Davidson, 1980). Therefore, if the employee's attention is directed to alcoholic beverage images, the responses indicate that the symbol position should be faster than those arising from its appearance in the position of the control images. In this task, the target and control images can be displayed at different exposure times. A high point of this task, unlike the Stroop task, is that it allows the measurement of different stages of attention, including implicit (automatic) and explicit (controlled) processes.

\section{Conclusion}

The purpose of this essay was to theoretically address possible relationships between frequency of participation in alcohol-prevention programs in the workplace and attentional bias to alcohol cues related to drinking. Having the dual-process model of information processing (Cohen et al., 1990; Moors \& De Houwer, 2006) and the mere-exposure effect model (Zajonc, 1968) as background theories, this paper emphasized the benefits of using implicit measures in HRM aimed at controlling alcohol misuse in the workplace. The discussion was primarily based on previously methodological suggestions made in a major HRM journal (Haines \& Sumner, 2013) and on tested procedures (Xu et al., 2014). Not all methodological rigors, or the ethical details that need to be presented in such assessments, were discussed in this review paper, which is a limitation. Another limitation is that we did not provide empirical proof for the proposed hypothesis. Future studies could test this assumption, therefore providing evidence to support, or not support, the measurement of attentional bias aimed at controlling alcohol misuse at work.

Despite these limitations, this essay proposes that HRM programs orientated to alcohol prevention in the workplace might have been oversimplified by a planned cognitive-processes orientation. Additionally, it makes a contribution to managers and scholars who wish to focus on new elements in these programs, such as implicit cognition. Although indirectly, it also stresses the benefits of using computerized tasks for the collection of implicit measures for HRM. It is worth noting that, in addition to measurements of attentional bias, specialized software can create, manage, and extract quantitative data from different cognitive-behavioral processes, such as implicit attitudes, decision making, and risk taking. Visual and audio stimuli software allows the recorded responses of participants with the aid of various devices, such as mouse, keyboard, and microphone (Stahl, 2006). Thus, it is possible to obtain quantitative data with relatively high temporal resolution and to make the statistical analysis important to the management. 


\section{References}

Aldana, S. G., Merrill, R. M., Price, K., Hardy, A., \& Hager, R. (2005). Financial impact of a comprehensive multisite workplace health promotion program. Preventive Medicine, 40(2), 131-137. https://doi.org/10.1016/j.ypmed.2004.05.008

Balsa, A. I., French, M. T., \& Regan, T. L. (2014). Relative Deprivation and Risky Behaviors. Journal of Human Resources, 49(2), 446-471. https://doi.org/10.1353/jhr.2014.0011

Barling, J., Dupre, K. E., \& Kelloway, E. K. (2009). Predicting Workplace Aggression and Violence Annual Review of Psychology (Vol. 60, pp. 671-692). Palo Alto: Annual Reviews.

Barsade, S. G., Ramarajan, L., \& Westen, D. (2009). Implicit affect in organizations. Research in Organizational Behavior, Vol 29: an Annual Series of Analytical Essays and Critical Reviews, 29, 135-162. https://doi.org/10.1016/j.riob.2009.06.008

Bazzani, L. C., \& Sanchez, A. I. M. (2016). Workplace Health Promotion: a path to follow. $\begin{array}{llll}\text { Ciência \& Saúde Coletiva, } & \text { 21(6), } & \text { 1909-1920. }\end{array}$ https://doi.org/10.1590/1413-81232015216.02522016

Becker, W. J., \& Menges, J. I. (2013). Biological implicit measures in HRM and OB: A question of how not if. Human Resource Management Review, 23(3), 219-228. https://doi.org/10.1016/j.hrmr.2012.12.003

Becker, W. J., Volk, S., \& Ward, M. K. (2015). Leveraging neuroscience for smarter approaches to workplace intelligence. Human Resource Management Review, 25(1), 56-67. https://doi.org/10.1016/j.hrmr.2014.09.008

Becker, William J., \& Cropanzano, Russell. (2010). Organizational neuroscience: The promise and prospects of an emerging discipline. Journal of Organizational Behavior, 31(7), 1055-1059. https://doi.org/10.1002/job.668

Becker, William J., Cropanzano, Russell, \& Sanfey, Alan G. (2011). Organizational Neuroscience: Taking Organizational Theory Inside the Neural Black Box. Journal of Management, 37(4), 933-961. https://doi.org/10.1177/0149206311398955

Block, N. (2005). Two neural correlates of consciousness. Trends in Cognitive Sciences, 9(2), 46-52. https://doi.org/10.1016/j.tics.2004.12.006

Bornstein, R. F. (1989). Exposure and affect - Overview and meta-analysis of research, 1968-1987. Psychological Bulletin, 265-289. https://doi.org/10.1037/0033-2909.106.2.265

Burns, C., Mearns, K., \& McGeorge, P. (2006). Explicit and implicit trust within safety culture. Risk Analysis, 26(5), 1139-1150. https://doi.org/10.1038/nprot.2006.35

Bush, G., Whalen, P. J., Shin, L. M., \& Rauch, S. L. (2006). The counting Stroop: a cognitive interference task. Nature Protocols, 1(1), 230-233. 
Byrne, A., Dionisi, A. M., Barling, J., Akers, A., Robertson, J., Lys, R., . . Dupre, K. (2014). The depleted leader: The influence of leaders' diminished psychological resources on leadership behaviors. Leadership Quarterly, 25(2), 344-357. https://doi.org/10.1016/j.leaqua.2013.09.003

Cabecas, J. M. M. (2015). Taxonomy to characterize occupational hazards (risk factors) at the workplace level. Work-a Journal of Prevention Assessment \& Rehabilitation, 51(4), 703-713. https://doi.org/10.3233/WOR-152023

Cohen, J. D., Dunbar, K., \& McClelland, J. L. (1990). On The Control of Automatic Processes - A Parallel Distribuited-Processing Account of The Stroop Effect Psychological Review, 97(3), 332-361. https://doi.org/10.1037//0033-295X.97.3.332

Cox, W. M., Brown, M. A., \& Rowlands, L. J. (2003). The effects of alcohol cue exposure on non-dependent drinkers' attentional bias for alcohol-related stimuli. Alcohol and Alcoholism, 38(1), 45-49. https://doi.org/10.1093/alcalc/agg010

Cox, W. M., Fadardi, J. S., \& Pothos, E. M. (2006). The alcohol Stroop test: A measure of attentional bias. Alcoholism-Clinical and Experimental Research, 30(9), 104A-104A.

Cropanzano, Russell, \& Becker, William J. (2013). The Promise and Peril of Organizational Neuroscience: Today and Tomorrow. Journal of Management Inquiry, 22(3), 306-310. https://doi.org/10.1177/1056492613478518

Cunningham, W. A., Zelazo, P. D., Packer, D. J., \& Van Bavel, J. J. (2007). The iterative reprocessing model: A multilevel framework for attitudes and evaluation. Social Cognition, 25(5), 736-760. https://doi.org/10.1521/soco.2007.25.5.736

Dager, Alecia D., Anderson, Beth M., Rosen, Rivkah, Khadka, Sabin, Sawyer, Broderick, Jiantonio-Kelly, Rachel E., . . Pearlson, Godfrey D. (2014). Functional magnetic resonance imaging (fMRI) response to alcohol pictures predicts subsequent transition to heavy drinking in college students. Addiction, 109(4), 585-595. https://doi.org/10.1111/add.12437

Deitz, D., Cook, R., \& Hersch, R. (2005). Workplace health promotion and utilization of health services - Follow-up data findings. Journal of Behavioral Health Services \& Research, 32(3), 306-319. https://doi.org/10.1111/add.12437

di Pellegrino, Giuseppe, Magarelli, Silvia, \& Mengarelli, Flavia. (2011). Food pleasantness affects visual selective attention. Quarterly Journal of Experimental Psychology, 64(3), 560-571. https://doi.org/10.1080/17470218.2010.504031

Eliason, M., \& Storrie, D. (2009). Does Job Loss Shorten Life? Journal of Human Resources, 44(2), 277-302. https://doi.org/10.3368/jhr.44.2.277

Field, Matt, \& Cox, W. Miles. (2008). Attentional bias in addictive behaviors: A review of its development, causes, and consequences. Drug and Alcohol Dependence, 97(1-2), 1-20. https://doi.org/10.1016/j.drugalcdep.2008.03.030 
Field, Matt, Munafo, Marcus R., \& Franken, Ingmar H. A. (2009). A meta-analytic investigation of the relationship between attentional bias and subjective craving in substance abuse. Psychological Bulletin, 135(4), 589-607. https://doi.org/10.1037/a0015843

Field, Matt, \& Quigley, Martin. (2009). Mild Stress Increases Attentional Bias in Social Drinkers Who Drink to Cope: A Replication and Extension. Experimental and Clinical Psychopharmacology, 17(5). https://doi.org/10.1037/a0017090

Frone, M. R. (2016). The Great Recession and Employee Alcohol Use: A US Population Study. Psychology of Addictive Behaviors, 30(2), 158-167. https://doi.org/10.1037/adb0000143

Gedro, J., Mercer, F., \& Iodice, J. D. (2012). Recovered alcoholics and career development: Implications for human resource development. Human Resource Development Quarterly, 23(1), 129-132. https://doi.org/10.1002/hrdq.21118

Haines, E. L., \& Sumner, K. E. (2013). Digging deeper or piling it higher? Implicit measurement in organizational behavior and human resource management. Human Resource Management Review, 23(3), 229-241. https://doi.org/10.1016/j.hrmr.2012.12.004

Hepworth, R., Mogg, K., Brignell, C., \& Bradley, B. P. (2010). Negative mood increases selective attention to food cues and subjective appetite. Appetite, 54(1), 134-142. https://doi.org/10.1016/j.appet.2009.09.019

Hofmann, Wilhelm, Gschwendner, Tobias, Friese, Malte, Wiers, Reinout W., \& Schmitt, Manfred. (2008). Working memory capacity and self-regulatory behavior: Toward an individual differences perspective on behavior determination by automatic versus controlled processes. Journal of Personality and Social Psychology, 95(4), 962-977. https://doi.org/10.1037/a0012705

Huang, Y. , \& Hsieh, P. (2013). The mere exposure effect is modulated by selective attention but not visual awareness. Vision Research, 91, 56-61. https://doi.org/10.1016/j.visres.2013.07.017

Li, Hai-Jiang, Yang, Juan, Jia, Lei, \& Zhang, Qing-Lin. (2011). Attentional bias in individuals with different level of self-esteem. [Attentional bias in individuals with different level of self-esteem.]. Acta Psychologica Sinica, 43(8), 907-916.

Lopes, F. M., Viacava, K. R., \& Bizarro, L. (2015). Attentional bias modification based on visual probe task: Methodological issues, results and clinical relevance. Trends in Psychiatry and Psychotherapy, 37(4), 183-193. https://doi.org/10.1590/2237-6089-2015-0011

Macleod, C., Mathews, A., \& Tata, P. (1986). Attentional bias in emotional disorders. Journal of Abnormal Psychology, 95(1), 15-20. https://doi.org/10.1037//0021-843X.95.1.15

Marquardt, N., Gades, R., \& Robelski, S. (2012). Implicit social cognition and safety culture. Human Factors and Ergonomics in Manufacturing \& Service Industries, 22(3), 213-234. https://doi.org/10.1002/hfm.20264 
Marteau, T. M., Hollands, G. J., \& Fletcher, P. C. (2012). Changing Human Behavior to Prevent Disease: The Importance of Targeting Automatic Processes. Science, 337(6101), 1492-1495. https://doi.org/10.1126/science.1226918

Molesworth, B. R. C., \& Chang, B. (2009). Predicting Pilots' Risk-Taking Behavior Through an Implicit Association Test. Human Factors, 51(6), 845-857. https://doi.org/10.1177/0018720809357756

Moors, A., \& De Houwer, J. (2006). Automaticity: A theoretical and conceptual analysis. Psychological Bulletin, 132(2), 297-326. https://doi.org/10.1037/0033-2909.132.2.297

Navon, D., \& Margalit, B. (1983). Allocation of attention according to informativeness in visual recognition. Quarterly Journal of Experimental Psychology Section a-Human Experimental Psychology, 35(AUG), 497-512. https://doi.org/10.1080/14640748308402484

Nigatu, Y. T., Reijneveld, S. A., Penninx, Bwjh, Schoevers, R. A., \& Bultmann, U. (2015). The Longitudinal Joint Effect of Obesity and Major Depression on Work Performance Impairment. American Journal of Public Health, 105(5), E80-E86. https://doi.org/10.2105/AJPH.2015.302557

Posner, M. I., Snyder, C. R. R., \& Davidson, B. J. (1980). Attention and the detection of signals. Journal of Experimental Psychology-General, 109(2), 160-174. https://doi.org/10.1037//0096-3445.109.2.160

Robbins, S. J., \& Ehrman, R. N. (2004). The role of attentional bias in substance abuse. Behav Cogn Neurosci Rev, 3(4), 243-260. https://doi.org/10.1177/1534582305275423

Robinson, B. M. , \& Elias, L. J. (2005). Novel stimuli are negative stimuli: Evidence that negative affect is reduced in the mere exposure effect. Perceptual and Motor Skills, 100(2), 365-372. https://doi.org/10.2466/pms.100.2.365-372

Robinson, T. E., \& Berridge, K. C. (1993). The neural basis of drug craving - an incentive-sensitization theory of addiction Brain Research Reviews, 18(3), 247-291. https://doi.org/10.1016/0165-0173(93)90013-P

Roche, A. M., Lee, N. K., Battams, S., Fischer, J. A., Cameron, J., \& McEntee, A. (2015). Alcohol use among workers in male-dominated industries: A systematic review of risk factors. Safety Science, 78, 124-141. https://doi.org/10.1016/j.ssci.2015.04.007

Rongen, Anne, Robroek, Suzan J. W., van Lenthe, Frank J., \& Burdorf, Alex. (2013). Workplace Health Promotion A Meta-Analysis of Effectiveness. American Journal of Preventive Medicine, 44(4), 406-415. https://doi.org/10.1016/j.amepre.2012.12.007

Spicer, R. S., \& Miller, T. R. (2016). The Evaluation of a Workplace Program to Prevent Substance Abuse: Challenges and Findings. Journal of Primary Prevention, 37(4), 329-343. https://doi.org/10.1007/s10935-016-0434-7

Stahl, C. (2006). Software for generating psychological experiments. Experimental Psychology, 53(3), 218-232. https://doi.org/10.1027/1618-3169.53.3.218 
Stormark, K. M., Laberg, J. C., Nordby, H., \& Hugdahl, K. (2000). Alcoholics' selective attention to alcohol stimuli: Automated processing? Journal of Studies on Alcohol, 61(1), 18-23. https://doi.org/10.15288/jsa.2000.61.18

Stroop, J. R. (1992). Studies of Interference in Serial Verbal Reactions (Reprinted from Journal of Experimental-Psychology, Vol 18, 643-662, 1935). Journal of Experimental Psychology-General, 121(1), 15-23. https://doi.org/10.1037/0096-3445.121.1.15

Tinghog, M. E. (2014). The workplace as an arena for universal alcohol prevention - what can we expect? An evaluation of a short educational intervention. Work-a Journal of Prevention Assessment \& Rehabilitation, 47(4), 543-551.

Tinghog, M. E., \& Tinghog, P. (2016). Preventing alcohol problems and improving drinking habits among employees: An evaluation of alcohol education. Work-a Journal of Prevention Assessment \& Rehabilitation, 53(2), 421-428. https://doi.org/10.3233/WOR-152231

Viacava, K. R., Weydmann, G. J., Tietze, A. W., Santolim, R. R., \& Bizarro, L. (2016). Attentional bias for food images after exposure to food commercials on TV. Journal of Food \& Nutritional Disorders, 5(6), 2-8. https://doi.org/10.4172/2324-9323.1000204

Whalen, Paul J., Bush, George, Shin, Lisa M., \& Rauch, Scott L. (2006). The emotional counting Stroop: a task for assessing emotional interference during brain imaging. Nature Protocols, 1(1), 293-296. https://doi.org/10.1038/nprot.2006.45

Williams, J. M. G., Mathews, A., \& MacLeod, C. (1996). The emotional stroop task and $\begin{array}{llll}\text { psychopathology. } & \text { Psychological } & \text { Bulletin, } & \text { 320(1), }\end{array}$ https://doi.org/10.1037/0033-2909.120.1.3

Xu, Y. S., Li, Y. J., Wang, G. X., Yuan, X., Ding, W. D., \& Shen, Z. X. (2014). Attentional bias toward safety predicts safety behaviors. Accident Analysis and Prevention, 71, 144-153. https://doi.org/10.1016/j.aap.2014.05.013

Yagi, Y., Ikoma, S., \& Kikuchi, T. (2009). Attentional Modulation of the Mere Exposure Effect. Journal of Experimental Psychology-Learning Memory and Cognition, 35(6), 1403-1410. https://doi.org/10.1037/a0017396

Zajonc, R. B. (1968). Attitudinal Effects of Mere Exposure. Journal of Personality and Social Psychology, 9(2P2), 1-\&. https://doi.org/10.1037/h0025848 